University of Nebraska - Lincoln

DigitalCommons@University of Nebraska - Lincoln

2013

Spectral distribution of wave energy dissipation by salt marsh vegetation

\author{
Ranjit S. Jadhav \\ Louisiana State University, rjadha1@lsu.edu \\ Qin Chen \\ Louisiana State University, qchen@lsu.edu \\ Jane M. Smith \\ Coastal and Hydraulics Laboratory, Jane.M.Smith@usace.army.mil
}

Follow this and additional works at: https://digitalcommons.unl.edu/usarmyresearch

Jadhav, Ranjit S.; Chen, Qin; and Smith, Jane M., "Spectral distribution of wave energy dissipation by salt marsh vegetation" (2013). US Army Research. 199.

https://digitalcommons.unl.edu/usarmyresearch/199

This Article is brought to you for free and open access by the U.S. Department of Defense at DigitalCommons@University of Nebraska - Lincoln. It has been accepted for inclusion in US Army Research by an authorized administrator of DigitalCommons@University of Nebraska - Lincoln. 


\title{
Spectral distribution of wave energy dissipation by salt marsh vegetation
}

\author{
Ranjit S. Jadhav ${ }^{\mathrm{a}}$, Qin Chen ${ }^{\mathrm{a}, *}$, Jane M. Smith ${ }^{\mathrm{b}}$ \\ a Department of Civil and Environmental Engineering, and Center for Computation and Technology, Louisiana State University, 3418 Patrick F. Taylor Hall, Baton Rouge, LA 70803, USA \\ ${ }^{\mathrm{b}}$ US Army Engineering Research and Development Center, Coastal and Hydraulics Laboratory, 3909 Halls Ferry Road, Vicksburg, MS 39180, USA
}

\section{A R T I C L E I N F O}

Article history:

Received 23 September 2012

Received in revised form 15 February 2013

Accepted 19 February 2013

Available online 27 March 2013

\section{Keywords:}

Drag coefficient

Drag force

Frequency-dependent energy dissipation

Random waves

Salt marsh vegetation

Wave damping

\begin{abstract}
A B S T R A C T
Spectral energy dissipation of random waves due to salt marsh vegetation (Spartina alterniflora) was analyzed using field data collected during a tropical storm. Wave data (significant wave heights up to $0.4 \mathrm{~m}$ in $0.8 \mathrm{~m}$ depth) were measured over a two-day period along a $28 \mathrm{~m}$ transect using 3 pressure transducers. The storm produced largely bimodal spectra on the wetland, consisting of low-frequency swell (7-10 s) and high-frequency (2-4.5 s) wind-sea. The energy dissipation varied across the frequency scales with the largest magnitude observed near the spectral peaks, above which the dissipation gradually decreased. The wind-sea energy dissipated largely in the leading section of the instrument array in the wetland, but the low-frequency swell propagated to the subsequent section with limited energy loss. Across a spectrum, dissipation did not linearly follow incident energy, and the degree of non-linearity varied with the dominant wave frequency. A rigid-type vegetation model was used to estimate the frequency-dependent bulk drag coefficient. For a given spectrum, this drag coefficient increased gradually up to the peak frequency and remained generally at a stable value at the higher frequencies. This spectral variation was parameterized by employing a frequency-dependent velocity attenuation parameter inside the canopy. This parameter had much less variability among incident wave conditions, compared to the variability of the bulk drag coefficient, allowing its standardization into a single, frequency-dependent curve for velocity attenuation inside a canopy. It is demonstrated that the spectral drag coefficient predicts the frequency-dependent energy dissipation with more accuracy than the integral coefficient.
\end{abstract}

(c) 2013 Elsevier B.V. All rights reserved.

\section{Introduction}

Wave propagation through vegetation is an important physical process along many coastal regions of the world, and along the shores of large inland lakes. Waves approaching vegetated shores lose energy due to obstructing vegetation. This reduces shore erosion and is of engineering significance for shoreline protection. The role and importance of coastal wetlands as a natural defense system against storm waves is generally acknowledged (e.g., Costanza et al., 2008; Dixon et al., 1998; Gedan et al., 2011; Lopez, 2009). Utilization of coastal wetlands to augment structural measures for mitigation of coastal flooding due to storm surge and waves is promoted in several regions of the world (e.g., Borsje et al., 2011; CPRA, 2012).

A body of literature exists quantifying reduction rates of integral wave heights due to vegetation (for summary, see Anderson et al., 2011; Jadhav and Chen, in review). Theoretical models based on energy conservation, have been proposed for application to both

\footnotetext{
* Corresponding author. Tel.: +1 225578 4911; fax: +1 2255784945 .

E-mail addresses: rjadha1@lsu.edu (R.S. Jadhav), qchen@lsu.edu (Q. Chen), Jane.M.Smith@usace.army.mil (J.M. Smith).
}

monochromatic waves (Dalrymple et al., 1984), and for narrowbanded random waves (Mendez and Losada, 2004). Kobayashi et al. (1993) presented an approach based on continuity and momentum equations, which assumed an exponential decay of integral wave height. Chen and Zhao (2012) proposed a vegetation-induced dissipation model based on the formulation of Hasselmann and Collins (1968) for energy dissipation of random waves by bottom friction. All these models assume rigid vegetation. A number of recent studies have underscored the importance of accounting for the stem and blade motion of flexible vegetation, and have proposed models that account for it (Bradley and Houser, 2009; Mullarney and Henderson, 2010; Riffe et al., 2011). Wave attenuation has been studied in a controlled laboratory environment (Augustin et al., 2009; Dubi and Tørum, 1996; Løvås and Tørum, 2001; Stratigaki et al., 2011), in field conditions involving salt marshes (Bradley and Houser, 2009; Cooper, 2005; Jadhav and Chen, in review; Möller, 2006; Möller and Spencer, 2002; Möller et al., 1999; Riffe et al., 2011), coastal mangrove forests (Mazda et al., 2006; Quartel et al., 2007), and vegetated lakeshores (Lövstedt and Larson, 2010). Most of these studies primarily focused on the attenuation of integral wave heights or wave energy, and estimation of integral bulk vegetation drag coefficients. As a step beyond integral dissipation characteristics, Lowe et al. (2005) developed an analytical model to predict the magnitude of the in-canopy velocity of 
waves propagating over a model canopy made up of rigid cylinders. Lowe et al. (2007) extended this model to random waves and predicted that high frequency components of wave energy would dissipate more efficiently inside the canopy. The model was verified with measurements taken from an artificial rigid cylinder canopy submerged on a barrier reef (random wave conditions) for $2 \mathrm{~h}$ and assuming a constant drag coefficient.

In the case of natural vegetation under random waves generated by a tropical cyclone, there are no published studies that examine in detail the frequency-based characteristics of wave energy dissipation and drag coefficient, though some studies have illustrated such characteristics with an example (Bradley and Houser, 2009; Paul and Amos, 2011). The present study investigates the spectral characteristics of wave energy dissipation due to natural vegetation, and the relationship between dissipation and the incident wave energy spectrum, using comprehensive field data. The study also identifies spectral variation of the vegetation drag coefficient. We hypothesize that the frequencyvarying spectral drag coefficient will predict spectral distribution of energy dissipation more accurately than an integral drag coefficient. To test the hypothesis, a new method is developed to parameterize the spectral drag coefficient over the entire range of measured wave spectra. The spectral and integral drag coefficients are then both used to estimate energy dissipation losses, and these estimates are compared to the observed dissipation to assess the validity of the hypothesis.

The following section describes the spectral energy dissipation model proposed by Chen and Zhao (2012) which is used to estimate drag coefficients and introduces the velocity attenuation factor. Sections 3 and 4 describe the field program and the wave conditions. Section 5 contains data analysis, where spectral characteristics of the observed energy dissipation are examined. In Section 6, spectral variation of estimated drag coefficient is demonstrated, and the spectral behavior of the mean velocity attenuation parameter is quantified. The mean velocity attenuation parameter and average drag coefficients are then applied to predict energy dissipation and compared with the existing prediction methods in Section 7. Finally the results are discussed, followed by a summary and conclusions.

\section{Spectral energy dissipation model}

Assuming the linear wave theory holds, the evolution of normallyincident random waves propagating through vegetation can be expressed with the following wave energy balance equation,

$\frac{\Delta\left(E_{j} C_{g, j}\right)}{\Delta x}=-S_{d s, j}$

where subscript $j$ represents the $j$ th frequency component of a wave spectrum, $E$ is the spectral wave energy density, $C_{g}=n c$ is the group velocity, $c=\sqrt{(g / k) \tanh (k h)}$ is the phase speed, $k$ is the wave number, $h$ is the still water depth, $g$ is the acceleration due to gravity and coefficient $n$ is given by $n=(1 / 2)[1+(2 k h / \sinh 2 k h)]$. The cross-shore coordinate is given by $x$ pointing landward and $S_{d s}$ is the energy dissipation due to vegetation per unit horizontal area. All other source terms are considered negligible compared to the vegetation induced losses.

The spectral wave energy dissipation due to vegetation is obtained by using a reorganized form of the model proposed by Chen and Zhao (2012). Their model treats vegetation as rigid, cylindrical elements that impart drag forces on the flow. Further, only the drag forces due to pressure differences are considered, as they are much larger than those arising from friction in the hydraulic regimes encountered in the field conditions.
In this model, the spectral energy dissipation due to vegetation is expressed by,

$S_{d s, j}=\frac{1}{2} \frac{C_{D, j} b_{v} N_{v}}{g}\left(\frac{\sigma_{j}}{\sinh k_{j} h}\right)^{2}\left(\sum_{-h}^{-h+s h} U_{z, r m s}(z) \cosh ^{2}\left[k_{j}(h+z) \Delta z\right]\right) E_{j}$

where $C_{D j}$ is a bulk drag coefficient, $b_{v}$ is the stem diameter, $N_{v}$ is the vegetation population density, $\sigma_{j}$ is the wave angular frequency, $s$ is the ratio of vegetation height, $h_{v}$, to the still water depth, $h$, and $U_{r m s}$ is the root-mean-squared (RMS) velocity given by,

$U_{z, r m s}=\sqrt{2 \sum_{j=1}^{j=N_{f}} \frac{\sigma_{j}^{2} \cosh ^{2} k_{j}(h+z)}{\sinh ^{2} k_{j} h} E_{j} \Delta \sigma}$

where $N_{f}$ is the total number of frequency components of a spectrum.

Eq. (2) is based on the quadratic representation of the shear stress induced by the vegetation. We parameterize the shear stress due to vegetation drag at elevation $z$ (positive upwards with origin at the still water level) due to $j$ th component of the spectrum as,

$\tau_{z, j}=-\frac{1}{2} \rho b_{v} N_{v} C_{d} \alpha_{j} u_{z, j}\left|\alpha_{j} u_{z, j}\right| \Delta z$

where $\rho$ is the density of water and $\alpha_{j} u_{z, j}$ is the vegetation-affected velocity at elevation $z$, and $C_{d}$ is the drag coefficient corresponding to this velocity. The velocity attenuation parameter, $\alpha$, is defined as the ratio of the vegetation-affected velocity, $u_{z}^{\prime}$, to the velocity in the absence of vegetation, $u_{z}$, at elevation $z$ inside the canopy:

$\alpha_{z, j}=\frac{u_{z, j}^{\prime}}{u_{z, j}}$

This parameter is similar to Lowe et al. (2005) but not exactly the same.

Similar to the definition of $\alpha$ (Eq. (5)), the ratio of the vegetationaffected RMS velocity at an elevation $z, U_{z, r m s}^{\prime}$, to the RMS velocity in the absence of vegetation, $U_{z, r m s}$, at elevation $z$ inside the canopy is defined as,

$\alpha_{z, r}=\frac{u_{z, r m s}^{\prime}}{u_{z, r m s}}$

Using these definitions, Chen and Zhao (2012) formulation is reorganized and the spectral distribution of energy dissipation is expressed as,

$S_{d s, j}=\frac{1}{2} \frac{\bar{C}_{D} b_{v} N_{v}}{g} \frac{\alpha_{z, j}^{2}}{\alpha_{z, r}^{2}}\left(\frac{\sigma_{j}}{\sinh k_{j} h}\right)^{2}\left(\sum_{-h}^{-h+s h} U_{z, r m s} \cosh ^{2}\left[k_{j}(h+z)\right] \Delta z\right) E_{j}$

where $\bar{C}_{D}$ is the spectrally-averaged, or integral, drag coefficient. To facilitate solution of Eq. (7), $\alpha$ is assumed to be independent of depth, and a normalized form of $\alpha$ is introduced as,

$\alpha_{n, j}=\frac{\alpha_{j}}{\alpha_{r}}$

Note that while $\alpha_{j}$ is always less than $1, \alpha_{n j}$ can be greater than 1 . Using $\alpha_{n, j}$, Eq. (7) can then be re-written as,

$S_{d s, j}=\frac{1}{2} \frac{\bar{C}_{D} b_{v} N_{v}}{g} \alpha_{n, j}^{2}\left(\frac{\sigma_{j}}{\sinh k_{j} h}\right)^{2}\left(\sum_{-h}^{-h+s h} U_{z, r m s} \cosh ^{2}\left[k_{j}(h+z)\right] \Delta z\right) E_{j}$. 
The spectrally variable drag coefficient is then expressed as,

$C_{D, j}=\bar{C}_{D} \cdot \alpha_{n, j}^{2}$

Integrated over the entire spectrum, the time-averaged rate of energy dissipation per unit area is given by,

$S_{v}=\sum_{j=1}^{j=N_{f}} S_{d s, j} \Delta \sigma$

\section{Study area and field program}

The study site was a salt marsh wetland in Terrebonne Bay on the Louisiana coast of the Gulf of Mexico (Fig. 1) west of the Mississippi River bird-foot delta. The shallow (depth, 1-3 m), micro-tidal (diurnal tidal range $<0.5 \mathrm{~m}$ ) bay is bordered by salt marsh to the north, and a series of narrow, low-lying barrier islands to the south. The waves in the bay consist of frequent low-energy offshore swell and locally generated seas which intensify during the passages of annual winter cold fronts and tropical cyclones.

During Tropical Storm Lee (September 3-4, 2011), three wave gages (pressure transducers W1 through W3) were deployed on a vegetated platform marsh along a north-south transect ( $28 \mathrm{~m}$ long) approximately perpendicular to the salt marsh edge (Fig. 1). The shore-normal has a bearing of $20^{\circ}$. A maximum of $20^{\circ}$ error in the alignment of waves to the wave gage array would overestimate the travel distances between the gages by about $6 \%\left(1-\cos 20^{\circ}\right)$ introducing corresponding error in estimates of energy dissipation. Waves approached from the south and propagated from gage W1 to W3 through vegetation. Gage W1 was located more than $16 \mathrm{~m}$ inwards of the marsh edge to avoid the waves breaking at the marsh edge. The self-logging pressure sensors sampled continuously at $10 \mathrm{~Hz}$ over the 2-day duration of the storm.

The dominant vegetation at the site is Spartina alterniflora, having a thick stem and thin, tapering flexible narrow blades. The average measured vegetation properties were; $N_{v}=422 \mathrm{stems} / \mathrm{m}^{2}$, $h_{v}=0.22 \mathrm{~m}$ (stem height), $h_{v t}=0.63 \mathrm{~m}$ (total plant height), $b_{v}=8.0 \mathrm{~mm}$, and $E_{v}=80 \mathrm{MPa}\left(E_{v} I_{v}=0.015 \mathrm{~N}-\mathrm{m}^{2}\right)$ where $E_{v} I_{v}$ is the flexural rigidity and $I_{v}$ is the second moment of inertia of a stem. Jadhav and Chen (in review) show a small variability of the vegetation properties between W12 and W23, which has been considered in the data analysis. Based on our observations and the estimated non-dimensional stiffness parameter (Mullarney and Henderson, 2010), the vegetation can be treated as rigid (see analysis in Jadhav and Chen, in review).

The time series of continuous pressure measurement from wave gages were analyzed using standard spectral techniques (e.g., Bendat and Piersol, 2000). The resulting energy spectra had a bandwidth, $\Delta_{f}$, of $0.01 \mathrm{~Hz}$, with $95 \%$ of the spectral energy between 0.03 and $0.7 \mathrm{~Hz}$. Thus each spectrum had 69 frequency components ( $N_{f}$ in Eqs. (3) and (11)). The integral wave parameters are defined as: significant wave height, $H_{m o}=4 \sqrt{m_{0}}$; mean wave period, $T_{z}=\sqrt{m_{0} / m_{2}}$; and spectral width, $v=\left(m_{0} m_{2} / m_{1}^{2}-1\right)$ where $m_{0}, m_{1}$, and $m_{2}$ are the zero-th, first and second moment of the wave spectrum, respectively.

The wave energy loss due to vegetation was considered dominant compared to the other source terms. To ascertain the validity of this assumption, the relative magnitude of source terms for the local wave generation and the losses due to bottom-friction, white-capping, and depth-limited breaking were evaluated. The wave records with significant potential for the magnitude of these source terms to be dominant, were removed from further analysis (for details see Jadhav and Chen, in review).

\section{Overview of wave conditions}

A total of 177 wave records ( 59 records at each of the 3 gages) were analyzed in this study. Table 1 lists summary statistics of water depth, zero-moment wave height, mean period and some derived parameters characterizing the wave conditions. The statistics in Table 1 describe only the analyzed data, not the entire measured dataset. As stated in the previous section, the wave records that violated assumptions of Eq. (1) were removed from analysis. With the diurnal tide augmented by the storm surge, the water depth rose from about $0.1 \mathrm{~m}$ to $0.8 \mathrm{~m}$ and then fell along with the tide. Only the measurements collected when water depth was greater than $0.4 \mathrm{~m}$ were used in the analyses, because wave energy levels were insignificant when water depth was less than $0.4 \mathrm{~m}$.

The incident significant wave heights $\left(H_{m o}\right)$ on the marsh varied from 0.05 to $0.39 \mathrm{~m}$ and were directly proportional to the depth of flood water. The recorded wave spectra were largely bimodal (Fig. 2) with distinct low-frequency swell (7-10 s) and wind-sea components $(2-4.5 \mathrm{~s})$. The relative depth, $h / \mathrm{L} z$, was less than 0.2 indicating relatively shallow water depths during the observation period (wave length $L z$ is based on the mean wave period). Spectral width, $v$, ranged from 0.42 to 0.86 , an indicator of the broad nature of the observed spectra. Table 1 also shows the Ursell number, $H_{m o} L_{z}^{2} / h^{3}$, as a measure of non-linearity. Waves were largely non-linear at $\mathrm{W} 1$ but the non-linearity quickly reduced as the waves propagated further (beyond W2) into the marsh and were dissipated by the vegetation.

\section{Observed spectral wave energy dissipation characteristics}

Measured spectra showed significant wave energy reduction over vegetation, as evidenced by the reduction in wave heights (Table 1 ).

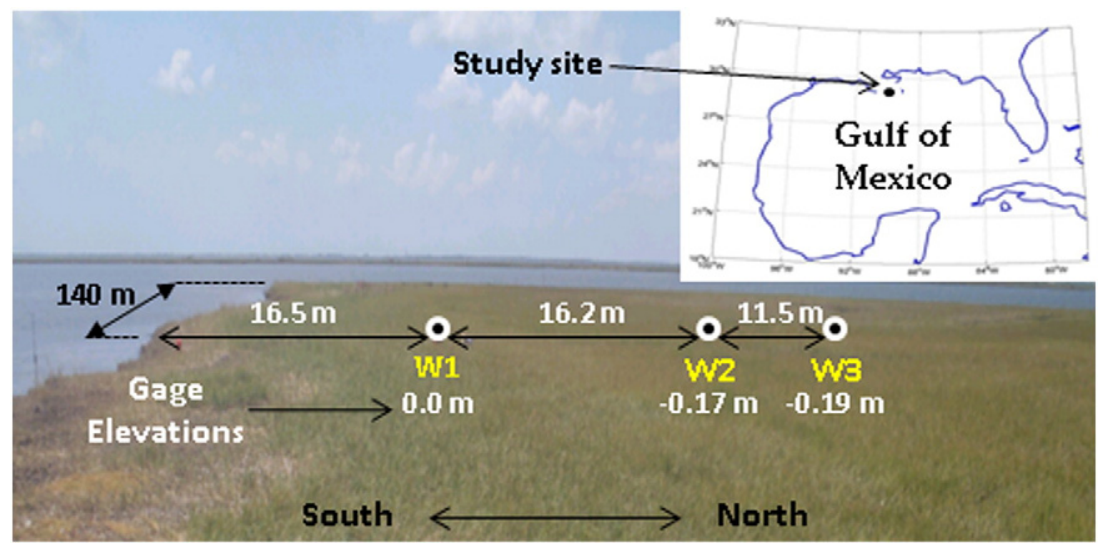

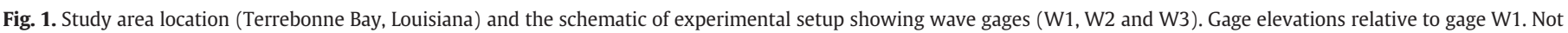
to scale. 
Table 1

Range and mean (in parentheses) values of analyzed wave parameters.

\begin{tabular}{llll}
\hline Parameter & Gage W1 & Gage W2 & Gage W3 \\
\hline Depth, $h(\mathrm{~m})$ & $0.40-0.82(0.55)$ & $0.57-1.0(0.72)$ & $0.57-1.01(0.72)$ \\
Significant wave height, & $0.15-0.40(0.24)$ & $0.07-0.28(0.14)$ & $0.04-0.21(0.09)$ \\
$\quad H_{m o}(\mathrm{~m})$ & & & \\
Peak wave period, $T_{p}(\mathrm{~s})$ & $2.5-4.7(4.0)$ & $1.2-4.5(2.3)$ & $1.3-4.5(2.6)$ \\
Relative wave height, & $0.36-0.49(0.41)$ & $0.12-0.29(0.18)$ & $0.08-0.22(0.12)$ \\
$H_{m o} / h$ & & & \\
Relative depth, $h / L_{z}$ & $0.07-0.13(0.10)$ & $0.09-0.16(0.13)$ & $0.10-0.16(0.12)$ \\
Spectral width, $v$ & $0.45-0.58(0.51)$ & $0.44-0.64(0.5)$ & $0.43-0.65(0.53)$ \\
Ursell number, $H_{m o} L_{z}^{2} / h^{3}$ & $29-81(48)$ & $9-16(11)$ & $6-10(8)$ \\
\hline
\end{tabular}

Energy reduction with respect to frequency was calculated between pairs of wave gages (W1-W2 and W2-W3) based on the measured wave energy density spectra, using Eq. (1). Ensemble averages of all analyzed energy density spectra, along with the ensemble average of the energy dissipation are shown in Fig. 3 for reaches W1-W2 (between gages W1 and W2) and W2-W3 (between gages W2 and W3). The energy density and dissipation are normalized by, $m_{0}$, the zero-th moment of the individual spectrum measured at the windward gage of the pair of gages bounding the reach. Fig. 3 shows that the magnitude of energy dissipation varies with the frequency. Higher dissipation was observed at the frequencies adjacent to the spectral peak in both reaches. Most of the wind-sea energy dissipated in the leading vegetation reach, W1-W2. Significant portions of swell energy propagated beyond the leading reach and dissipated in reach W2-W3.

Fig. 3 also shows that the dominant loss near the spectral peak is less pronounced in the second reach, W2-W3, where a substantial portion of the total energy loss occurs at frequencies higher than the peak. This is illustrated in Fig. 4 where energy reduction in the dominant wave frequencies, i.e., swell and wind-sea band $(0.03-0.36 \mathrm{~Hz})$, as a percentage of the total $(0.03-0.7 \mathrm{~Hz})$ energy reduction is plotted as a function of Keulegan-Carpenter number, $K_{C}$. The $K_{C}$ number is defined as, $K_{C}=U_{r m s} T_{z} / b_{v}$, where $U_{r m s}$ is the root-mean-square orbital velocity at the bed, considering the entire spectrum. In reach $\mathrm{W} 1-\mathrm{W} 2$, wave energy reduction in the swell and wind-sea bands accounted for 55 to $70 \%$ of the total reduction, while in reach W2-W3, this percentage was only 40 to $55 \%$. Thus, in reach W2-W3 the energy reduction was more evenly distributed between dominant and higher frequencies. This is partly due to modification of the spectral shape as a result of the non-linear transfer of energy to the higher frequencies as waves propagated from gage W1 to W2.

Across the frequencies above the peak, the spectral distribution of energy dissipation was observed to gradually taper off. The rate of such tapering with respect to spectral frequency is shown in Fig. 5 using normalized dissipation $\left(S_{d s}(f) / E(f)\right)$ for 3 ranges of $K_{C}$ numbers. The choice of the range of $K_{C}$ for ensemble averaging is inconsequential and is made for the purpose of creating three ranges of $K_{C}$ signifying ranges of hydrodynamic conditions. Variation of the frequency exponent over all spectra with respect to $K_{C}$ number is shown in Fig. 6. Larger $K_{C}$ numbers generally represent waves in reach W1-W2. Waves in this reach were more energetic, with more peaked spectra and larger concentration of energy in the swell-sea band $(0.03-0.36 \mathrm{~Hz})$. The smaller values of $K_{C}$ numbers represent relatively low energy waves with much broader spectra. Fig. 5 shows that at frequencies above the peak, and at higher $K_{C}$ numbers, the normalized energy dissipation has a stronger dependence on frequency.

The current standard modeling practice assumes that the distribution of energy dissipation generally follows the incident wave energy density spectrum (e.g., Suzuki et al., 2011). To assess the validity of this assumption, the following hypothesis was tested using our field study measurements:

$S_{d s}(f)=a \cdot E(f)^{b}$

where $a$ and $b$ are determined by regression analysis. For a given reach (W1-W2 or W2-W3), each incident energy spectrum, $E(f)$, and the corresponding dissipation spectrum, $S_{d s}(f)$, were divided into three frequency bands, representing swell $(0.03-0.16 \mathrm{~Hz})$, wind-sea $(0.16$ $0.32 \mathrm{~Hz}$ ) and high frequencies $(0.32-0.7 \mathrm{~Hz})$. These divisions correspond to the local spectral energy minima observed around $0.16 \mathrm{~Hz}$ and $0.32 \mathrm{~Hz}$ in the recorded bimodal spectra (Fig. 2). For each of these three frequency bands, a coefficient pair $(a, b)$ was determined by fitting Eq. (12) to the field data. Thus, for each spectrum (wave record), three coefficient pairs were obtained. Coefficient pairs where the fit of Eq. (12) to the field data resulted in an $R^{2}$ (coefficient of determination) less than 0.8 , were excluded from the analysis.

The exponent $b$ is a measure of linearity (linear when $b=1$ ) of the relationship between energy dissipation, $S_{d s}(f)$ and incident spectrum, $E(f)$. The probability of occurrence of $b$ is plotted in Fig. 7 for the three frequency bands, within three ranges of $K_{C}$ numbers. The $K_{C}$ number is based on the entire spectrum. Note that a $K_{C}$ value of about 60 segregates first pair of gages, W1-W2, and the second pair, W2-W3. Fig. 7 shows that the relationship between $S_{d s}(f)$ and $E(f)$ is not consistently linear $(b \neq 1)$ across the frequency scales. The relationship tends to be most linear in the wind-sea band across the entire range of $K_{C}$ numbers, with slightly narrowed distribution in the middle $K_{C}$ number range. The relationship between energy dissipation and incident spectrum becomes slightly more nonlinear in the swell frequency band. The coefficient $b$ tends to increase at smaller $K_{C}$ numbers (which are more common in the second reach, W2-W3). In
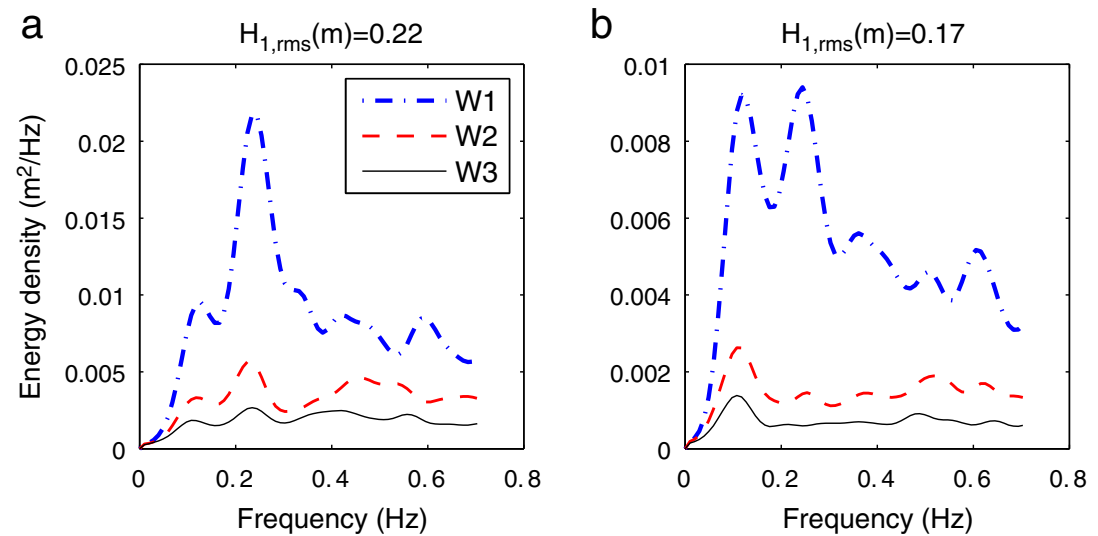

Fig. 2. Wave energy spectra recorded on September 3, 2011 at (a) 6:45 UTC and (b) 12:30 UTC. 
a

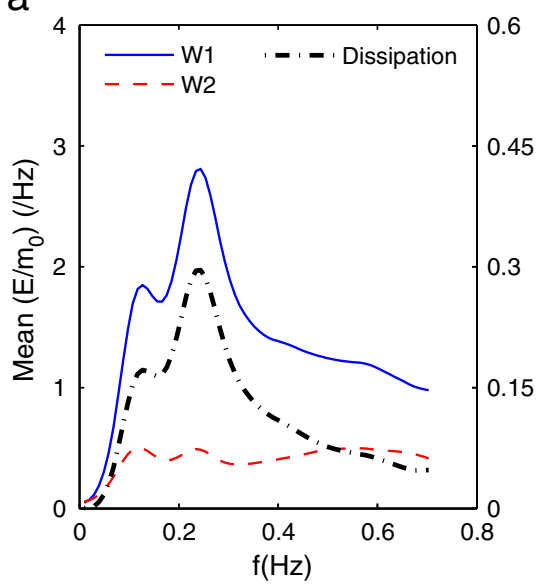

b

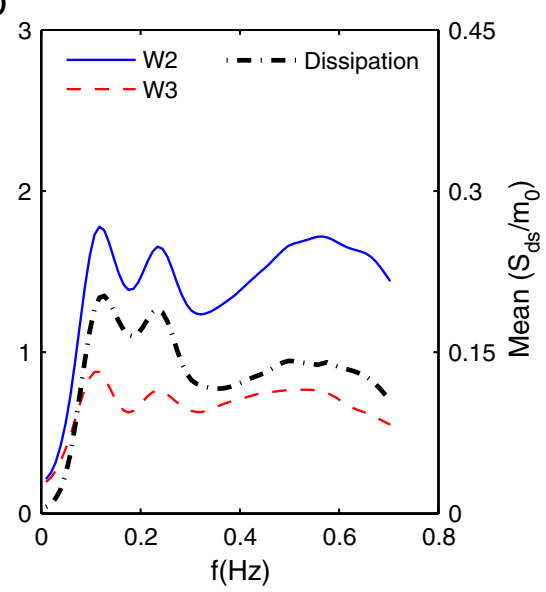

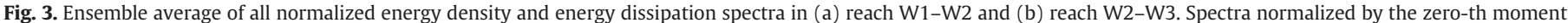
$\left(m_{0}\right)$ of the energy spectrum measured at the windward gage of the pair of gages.

the high-frequency band $\left(f>0.32 \mathrm{~Hz}\right.$ ) the relationship between $S_{d s}(f)$ and $E(f)$ is linear for waves with $K_{C}<47$, and gradually becomes nonlinear with increasing $K_{C}$ number. Note that the energy spectra and hence, the energy dissipation, in this high-frequency range is also affected by non-linear triad interactions.

Parameter $a$ in Eq. (12) was confirmed to be equal to the ratio of the integrated energy dissipation to the total wave energy, $S_{v} / m_{b}$, where $m_{b}=\int E^{b} d f$ and $S_{v}=\int S_{d s} d f$.

\section{Estimates of integral and frequency-dependent bulk drag coefficients}

The integral energy dissipation formulations (e.g., Mendez and Losada, 2004) assume the drag coefficient is independent of frequency and determine its single value, $\bar{C}_{D}$, for the entire spectrum, which is assumed to be narrow-banded. The variation of drag coefficient with the hydrodynamics has been typically related to the Reynolds $\left(R_{e}\right)$ and Keulegan-Carpenter $\left(K_{C}\right)$ numbers using empirical relationships. Several studies have developed empirical formulations for integral estimates of $\bar{C}_{D}$ (Bradley and Houser, 2009; Jadhav and Chen, in review; Kobayashi et al., 1993; Mendez and Losada, 2004; Mendez et al., 1999; Paul and Amos, 2011; Sánchez-González et al., 2011). The empirical relationships are a valuable tool for predicting integral wave

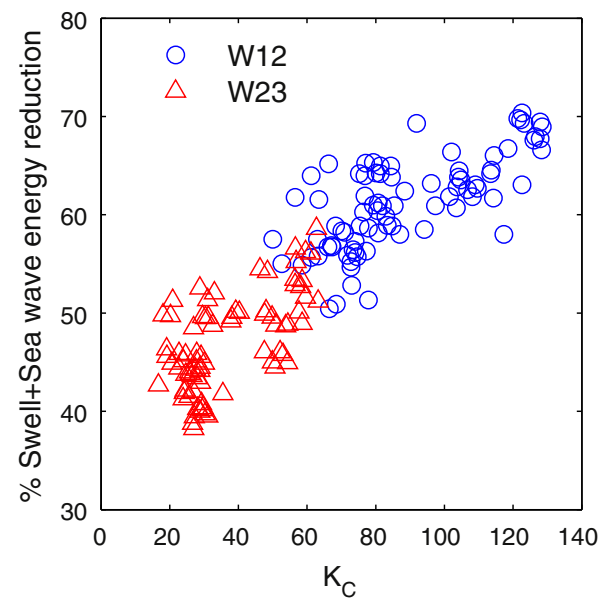

Fig. 4. Wave energy reduction in the swell and wind-sea band $(0.03-0.36 \mathrm{~Hz})$ as a percentage of the total $(0.03-0.7 \mathrm{~Hz})$ energy reduction. heights. For the data presented in this paper, the integral drag coefficients correlate well to the $K_{C}$ number $\left(R^{2}=0.95\right)$ (Fig. 8), resulting in the following empirical formula:

$\bar{C}_{D}=70 K_{C}^{-0.86} \quad 25<K_{C}<135$.

Note that this $\bar{C}_{D}$ represents the "bulk" value over the field study transect (vegetation patch), rather than the drag coefficient of an idealized, isolated, cylinder (e.g., Tanino and Nepf, 2008). The $\bar{C}_{D}$ in Fig. 8 was estimated using Eq. (2). Using the same equation, and allowing the drag coefficient to vary with frequency for each spectrum, produces a frequency distributed drag coefficient. Fig. 9 shows such distributions that are ensemble averaged over the three $K_{C}$ ranges. It is clear from these plots that the drag coefficient varies with the frequency, and a single integral drag coefficient value over the entire spectral frequency scale does not adequately represent the spectral evolution. This is most notable for the range containing the smallest $K_{C}$ numbers, where the drag coefficient varies by a factor of 6 . Therefore, in studies of wave spectral evolution dominated by energy losses due to vegetation, a spectrally varying drag coefficient will more accurately predict wave energy dissipation.

Eqs. (13) and (10) can be used to compute the frequency varying drag coefficient, $C_{D}$, when $\bar{C}_{D}$ and $\alpha_{n}$ are known. For a given spectrum (with its $K_{C}$ ), $\bar{C}_{D}$ can be determined using Eq. (13). To calculate $\alpha_{n}$, the following procedure was followed. Using the measured energy spectra, Eqs. (1) and (9) were numerically solved to compute $\alpha_{n j}$

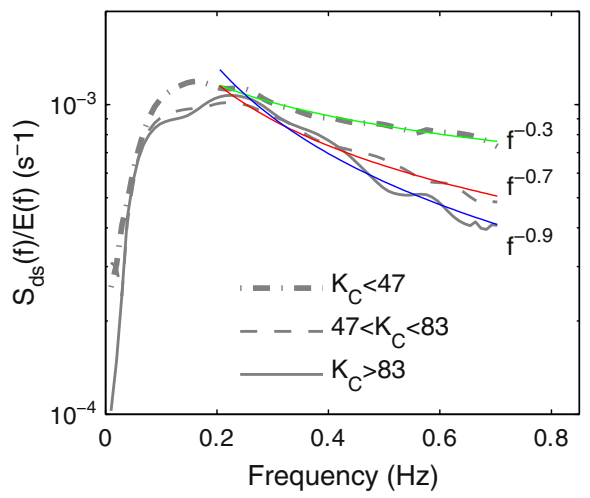

Fig. 5. Frequency distribution of the normalized energy dissipation rate. Curves represent ensemble averages of all measured spectra in reaches W1-W2 and W2-W3. The thin smooth solid lines represent a least-square fit to the data points above spectral peaks. 


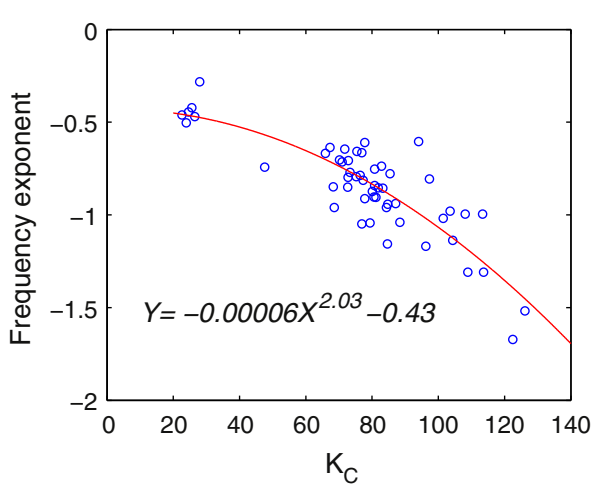

Fig. 6. Frequency exponent (from Fig. 5) versus Keulegan-Carpenter number for all spectra. Only data points with $R^{2}>0.8$ are shown.

for each frequency component of a spectrum. All $\alpha_{n, j}$ profiles were then ensemble-averaged, producing the single $\bar{\alpha}_{n}$ curve shown in Fig. 10. Across the spectrum of frequencies, $\bar{\alpha}_{n}$ gradually increases up to the region of the peak, and then slightly decreases. The $\bar{\alpha}_{n}$ values for frequencies above about $0.4 \mathrm{~Hz}$ are not considered reliable, due to the greater influence of non-linear energy transfer, and possible amplification of noise resulting from the pressure response function at those frequencies. The $\bar{\alpha}_{n}$ values for frequencies above $0.4 \mathrm{~Hz}$ are therefore excluded from the following analysis. Multiplying the integral $\bar{C}_{D}$ obtained from Eq. (13) by values of $\bar{\alpha}_{n}$ (Fig. 10), provides values that can be used in Eq. (10) to calculate frequency-dependent values of $C_{D}$, that can be used to predict the frequency-dependent energy dissipation in wave spectra.

\section{Prediction of energy dissipation using estimated drag coefficients}

To estimate energy dissipation due to vegetation in practical applications, selection of the appropriate drag coefficient is necessary. This section compares two approaches for selecting drag coefficients to determine which approach results in the better prediction of wave spectra in the presence of rigid-type vegetation. In the first, simple approach (existing standard practice), an integral drag coefficient, $\bar{C}_{D}$ (such as would be calculated using Eq. (13)) is specified and then spectral dissipation is calculated using Eq. (2). In the second approach, the frequency-dependent variable drag coefficient, $C_{D}$, is specified (Eq. (10)) and used in Eq. (2) to calculate spectral dissipation.

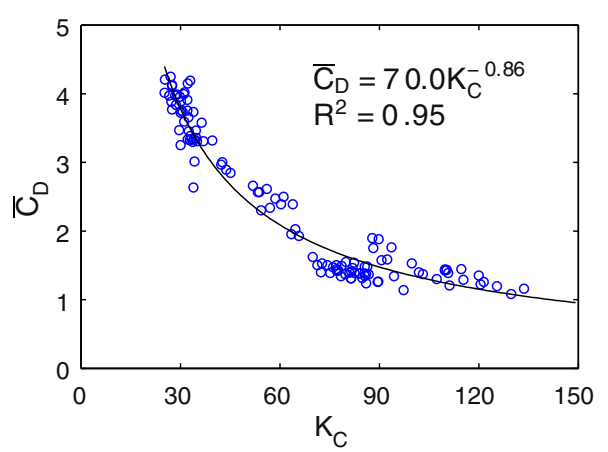

Fig. 8. Estimated integral bulk drag coefficient and its variation with the KeuleganCarpenter number.

Fig. 11 shows comparison plots of the measured and predicted energy dissipation using these two approaches, for one wave record. The frequency-dependent $C_{D}$ predicts the frequency distribution of energy dissipation with better accuracy than the integral $\bar{C}_{D}$. To quantitatively assess the predictive accuracy associated with the different approaches, over the entire dataset, the error between the measured and the predicted energy dissipation was calculated for each record and was ensemble averaged (Fig. 12a). In the frequency range with the dominant energy $(0.03-0.36 \mathrm{~Hz})$, the energy dissipation predicted by the frequency varying $C_{D}$ has much less error than that predicted by the integral $\bar{C}_{D}$. The improvement is especially significant in the vicinity of the spectral peak frequencies, where the largest dissipation is encountered. Additionally, Fig. 12b shows that, by employing the frequency-varying $C_{D}$, the model is able to predict total dissipation, $S_{v}$ (Eq. (11)) reliably. The error in the prediction of $S_{v}$ is generally less than $5 \%$. The mean error in the predicted $H_{m o}$ for the dominant frequency range $(0.03-0.36 \mathrm{~Hz})$ at gages $\mathrm{W} 2$ and $\mathrm{W} 3$ using the two methods $\left(\bar{C}_{D}\right.$ and $\left.C_{D}\right)$ are $(6.5 \%$ and $8.2 \%)$ and $(-5.0 \%$ and $-2.3 \%)$, respectively. At W2, the frequency-dependent $C_{D}$ method may appear slightly worse than the $\bar{C}_{D}$ method, however, the true advantage of the $C_{D}$ method is in the improved prediction of the frequency distribution of energy dissipation, as seen in Fig. 11a,b. This is reflected in the much better improvement in the estimate of mean period with errors being $(-9.0 \%$ and $4.1 \%)$ and $(-2.6 \%$ and $1.5 \%)$ at gages W2 and W3, respectively. Likewise the spectral width estimates are better when using $C_{D}$ compared to $\bar{C}_{D}$ with errors being $(-25.1 \%$ and $-5.4 \%)$ and $(-9.2 \%$ and $2.1 \%)$ at gages $\mathrm{W} 2$ and $\mathrm{W} 3$, respectively.
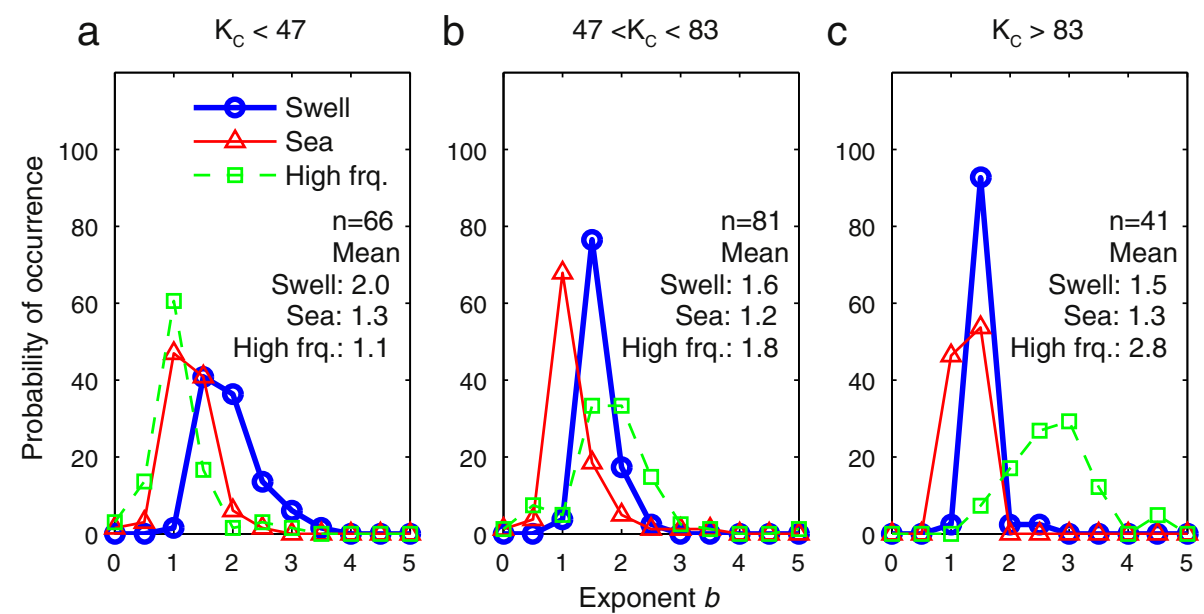

Fig. 7. Probability of occurrence of exponent $b$ (Eq. (12)) with respect to ranges of Keulegan-Carpenter number. 


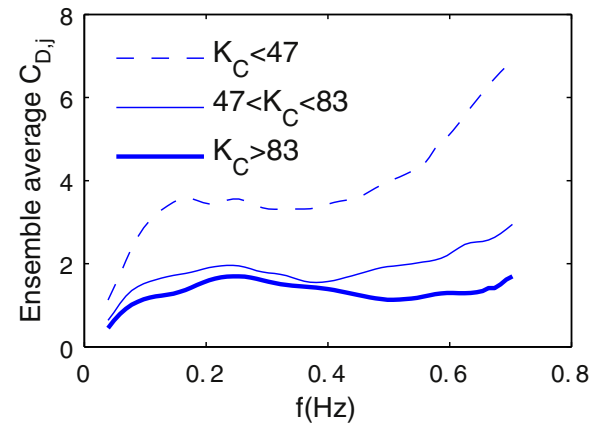

Fig. 9. Spectral variation of the bulk drag coefficient. All individual spectral distributions are ensembled averaged based on $K_{C}$ ranges.

\section{Discussion}

The Chen and Zhao (2012) formulation for energy dissipation through rigid vegetation has been reorganized by introducing the velocity attenuation parameter, $\alpha$. In this study, $\alpha$ is defined as the ratio of vegetation-attenuated orbital velocity inside the canopy at a given elevation, to the orbital velocity in the absence of vegetation at the same elevation. This is similar to the velocity attenuation parameter of Lowe et al. (2005), which was defined as the ratio of the velocity inside canopy to that above canopy. These two versions of the velocity attenuation parameter are related by a factor which results from the decay of orbital velocity with respect to depth. To illustrate the equivalence of these two parameters, $\alpha$ was calculated using the Tropical Storm Lee field data and compared to the velocity attenuation parameter values reported in Lowe et al. (2007, Fig. 5a). To this end, when calculating $\alpha$, the drag coefficient corresponding to the use of the velocity inside a canopy, Cd, was set to a fixed value of 2.5, as in Lowe et al. (2007). Fig. 8 shows that relatively stable value of the drag coefficient was observed for wave records with $K_{C}>85$, so only those wave records were used for this comparison. The values of $\alpha$ plotted in Fig. 13 are the result of ensemble averaging 118 (59 wave records at each of the 2 gages, W2 and W3) $\alpha$ profiles. Comparison of Fig. 13 with Fig. 5a of Lowe et al. (2007) shows that, in both cases, the velocity attenuation parameter decreases gradually over the longer waves with the maximum values associated with shorter period waves. The values of $\alpha$ associated with wave periods shorter than $2 \mathrm{~s}$ are less reliable due to the greater influence of non-linear energy transfers in that frequency band, and possible amplification of noise in the data analysis.

Because the formulations for energy dissipation given in Eqs. (8) and (9) are based on the velocities at the same elevation inside a canopy, the results can be applied to cases involving shallow water and emergent vegetation. Further, Eq. (9) consists of explicit integration over discrete vertical increments and can be conveniently adopted

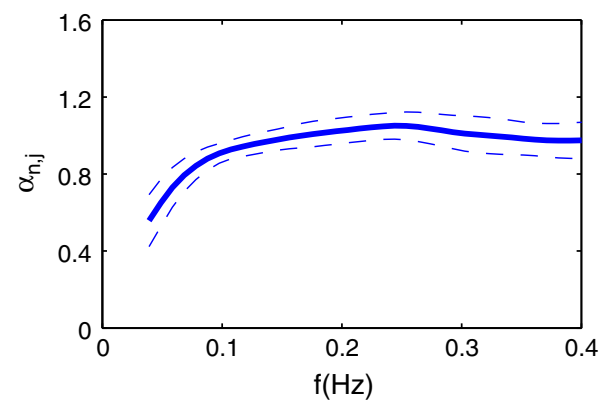

Fig. 10. Spectral variation of ensemble-averaged velocity attenuation parameter, $\alpha_{n}$, based on 118 spectra. Dashed lines represent \pm 1 standard deviation. when vertical variations of vegetation properties and hydrodynamics are important (e.g., Neumeier and Amos, 2006).

The velocity attenuation factor, $\alpha$, is directly proportional to the normalized energy dissipation $\left(S_{d s}(f) / \mathrm{E}(f)\right)$ as is evident from Eq. (7). In the special case of shallow water, this equation simplifies to,

$\alpha_{n}^{2} \propto \frac{S_{d s}}{E}$.

The equivalence of $\alpha_{n}^{2}$ and $S_{d s} / E$ is seen in the similarities between Figs. 5 and 10 in the dominant energy band. As shown in this study, the magnitude of the velocity attenuation factor is expected to decrease with increasing excursion (i.e., $K_{C}$ number). The lower $\alpha_{n}$ value reduces the normalized dissipation at the higher $K_{C}$ numbers in Fig. 5, causing a steeper decline of the frequency distributions as shown.

In the prediction of drag-induced energy dissipation, the drag coefficient is an important input parameter, and attempt to generalize it remains a challenge. Consistent estimates of drag coefficients based on a range of wave and vegetation conditions will improve predictability of $C_{D}$ as more data become available. Several complex processes are involved in the wave energy dissipation induced by vegetation drag. Laboratory studies of hydrodynamics around a single rigid circular cylinder in oscillating flows, in which force is modeled as a summation of inertial and drag forces by a Morison-type equation (Morison et al., 1950) contribute to understanding of these processes. Even in this simple form, under controlled conditions, the drag coefficients vary with time, Reynolds number, relative motion of the fluid, relative roughness, variable flow separation, wake interference, ambient turbulence, etc. (Sarpkaya, 1976). Additionally, in wavy flows (as opposed to simple oscillatory flows), velocity decays exponentially with depth and the orbital motion induces 3D flow effects and rotating vortices, further complicating the processes. Although Stokes' solution exists for force coefficients in un-separated and laminar oscillating flows, such information must be obtained using experimental studies for separated flows, which are present in the field conditions (Sarpkaya, 1976). In the case of natural vegetation, the necessity of deriving drag coefficients from field studies is underscored by the fact that, to effectively model field conditions, these coefficients need to represent a stem array rather than a single cylinder (Tanino and Nepf, 2008). If the vegetation is flexible, then the consideration of the stem motion becomes essential (Mullarney and Henderson, 2010).

\section{Summary and conclusions}

Random wave spectra were measured over salt marsh vegetation to study vegetation induced energy dissipation along a marsh transect with two reaches. The waves in the leading reach of the transect were more energetic, highly nonlinear, occurred in shallower water, and exhibited greater energy dissipation compared to the subsequent reach, where waves were less energetic, significantly less nonlinear, and exhibited less energy dissipation. Waves propagating over salt marsh vegetation dissipate energy due to drag induced by the stems. The magnitude of energy dissipation was observed to vary with the wave frequency. The greatest energy dissipation was observed near the incident spectral peak frequencies, with energy dissipation gradually decreasing with frequency above the peak. The rate of this decrease was greater for waves with larger $K_{C}$ numbers and lower for waves with decreasing $K_{C}$ numbers. Upon entering the vegetation, the low-frequency swell $(<0.16 \mathrm{~Hz})$ dissipated less in the leading reach of the measurement transect than the wind-sea $(0.16-0.32 \mathrm{~Hz})$, carrying energy further and continuing the dissipation process in the subsequent reach of the transect. On the other hand, the majority of the wind-sea energy dissipated in the leading reach of the transect. Across a spectrum, energy dissipation did not linearly follow incident energy density and the degree of non-linearity varied with the frequency scale. The relationship of the spectral dissipation to energy 

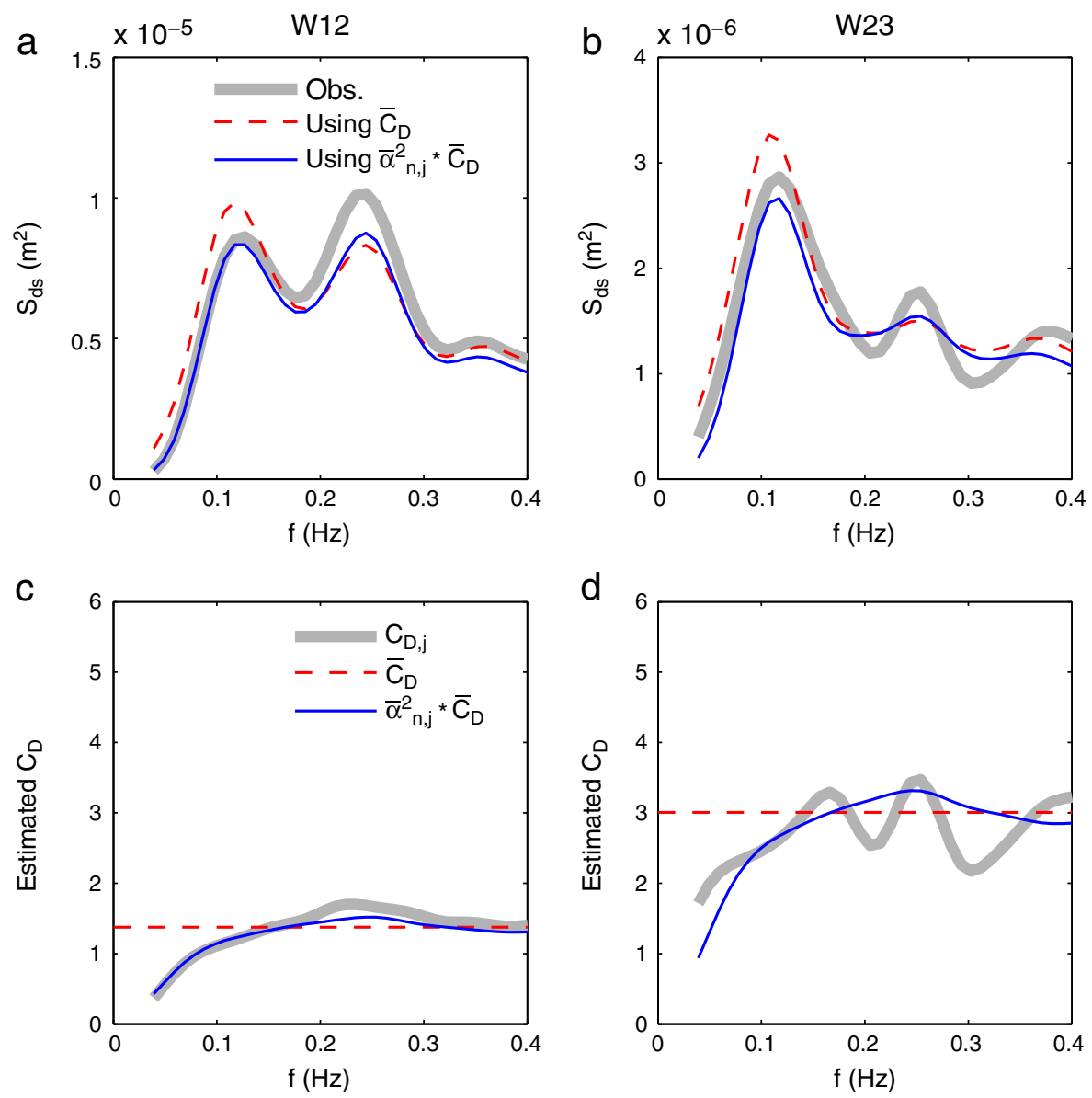

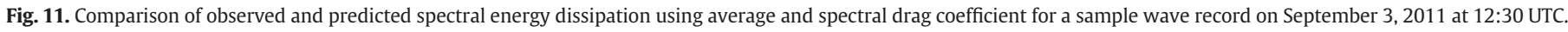
(a) Dissipation between W1-W2 and (b) dissipation between W2-W3. Dissipation based on $C_{D}$ values shown in (c) for W1-W2 and (d) for W2-W3.

density tended to be less nonlinear in the wind-sea than the swell band, but the relationship became slightly more nonlinear and consistent (across bands) for waves with larger $K_{C}$ numbers. In general, the relationship was slightly more nonlinear in the swell band than the wind-sea band.

The normalized wave energy dissipation $\left(S_{d s}(f) / E(f)\right)$ was observed to be greatest near the spectral peak frequencies. The magnitude of the normalized dissipation was directly related to the frequency in the band below the peak, and inversely related to the frequency in the band above the peak of the wave energy density spectrum.

The vegetation induced drag coefficient was shown to vary with frequency. The distribution increased gradually up to the spectral peak and then remained generally uniform. The magnitude of the peak of this distribution was directly related to the magnitude of the corresponding $K_{C}$ number of the waves. The frequency-dependent drag coefficient was parameterized by introducing a normalized
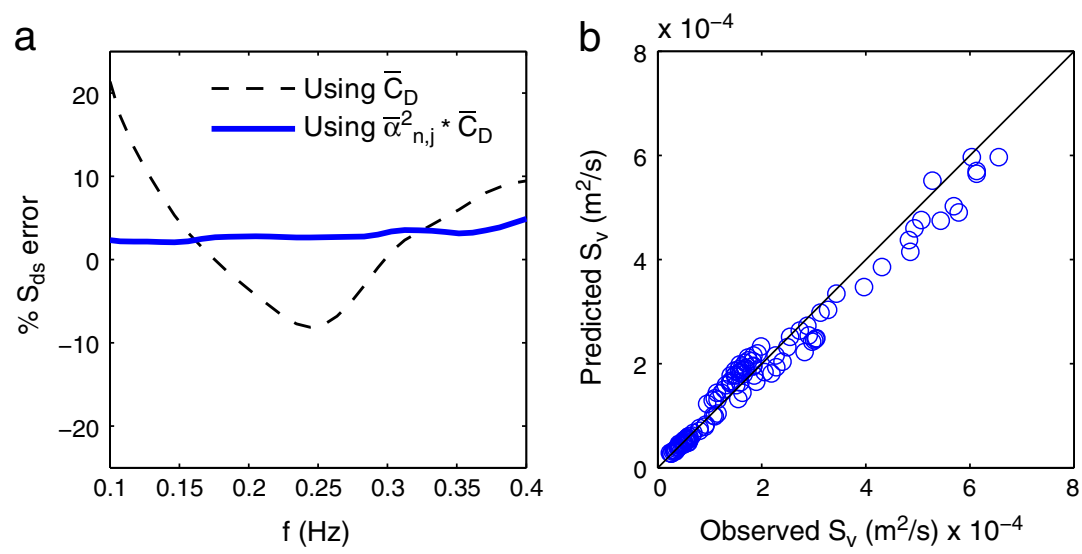

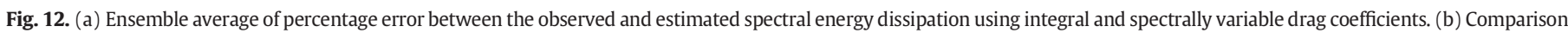
of predicted and observed total energy dissipation. 


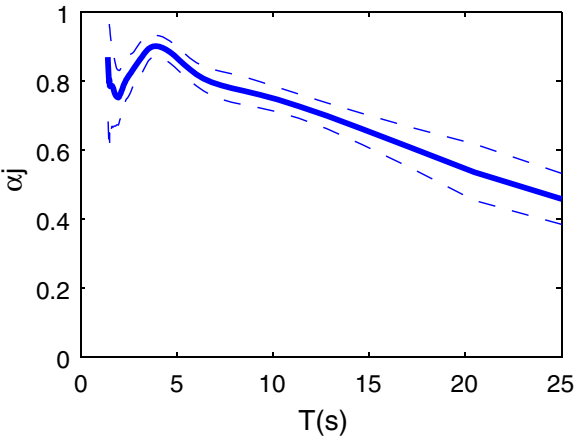

Fig. 13. Variation of ensemble-averaged $\alpha_{j}$ with wave period $T_{j}$. Dashed lines represent \pm 1 standard deviation.

velocity attenuation parameter, $\alpha_{n}$. The spectral profiles of $\alpha_{n}$ were ensemble-averaged and a single $\bar{\alpha}_{n}$ curve was developed. This single curve along with the integral drag coefficient allowed for a prediction of the frequency-dependent bulk drag coefficient. It was demonstrated that the frequency-dependent drag coefficient predicted the spectral distribution of energy dissipation with better accuracy than the integral drag coefficient.

The methodology and drag coefficient parameterization presented in this paper has been verified using the same dataset on which it is based. This validates the parameterization of the spectral bulk drag coefficient using a single velocity attenuation curve. This parameterization approach needs to be further tested using other, independent, datasets. Furthermore, accurately quantifying the cross-spectral energy transfer in the presence of vegetation is needed in order to understand the role of nonlinear triad interactions of shallow water waves in wave energy dissipation caused by vegetation. Effects of vegetation are being incorporated into a Boussinesq wave model and tested against the field data. Results will be reported on in the near future.

\section{Acknowledgments}

The study was supported by the US Department of Homeland Security (DHS) through the Southeast Region Research Initiative (SERRI) and by the US National Science Foundation (NSF) (Grant Nos. CBET-0652859 and DMS-1115527). We thank T. Baker Smith, LLC for the logistical support and topographic surveying, and the Louisiana Universities Marine Consortium (LUMCON) for providing meteorological data. Graduate students Kyle Parker, James Chatagnier and James Bouanchaud assisted in the field study. We also thank Dr. Weiming Wu of the University of Mississippi for helpful discussions. Any opinions, findings, conclusions and recommendations expressed in this paper are those of the authors and do not necessarily reflect the views of the NSF or the DHS.

\section{References}

Anderson, M.E., Smith, J.M., McKay, S.K., 2011. Wave dissipation by vegetation. Coasta and Hydraulics Engineering Technical Note ERDC/CHL CHETN-I-82.U.S. Army Engineer Research and Development Center, Vicksburg, MS.

Augustin, L.N., Irish, J.L., Lynett, P.J., 2009. Laboratory and numerical studies of wave damping by emergent and near-emergent wetland vegetation. Coastal Engineering 56, 332-340.

Bendat, J.S., Piersol, A.J., 2000. Random Data: Analysis and Measurement Procedures. John Wiley and Sons, Inc., New York, NY.

Borsje, B.W., van Wesenbeeck, B.K., Dekker, F., Paalvast, P., Bourma, T.J., van Katwijk, M.M. de Vries, M.M., 2011. How ecological engineering can serve in coastal protection. Ecological Engineering 37 (2), 113-122.

Bradley, K., Houser, C., 2009. Relative velocity of seagrass blades: implications for wave attenuation in low-energy environments. Journal of Geophysical Research 114, F01004. http://dx.doi.org/10.1029/2007JF000951.

Chen, Q., Zhao, H., 2012. Theoretical models for wave energy dissipation caused by vegetation. Journal of Engineering Mechanics 138 (2), 221-230.
Cooper, N.J., 2005. Wave dissipation across intertidal surfaces in the Wash Tidal inlet, Eastern England. Journal of Coastal Research 21 (1), 28-40.

Costanza, R., Pérez-Maqueo, O., Martinez, M.L., Sutton, P., Anderson, S.J., Mulder, K., 2008. The value of coastal wetlands for hurricane protection. Ambio 37, 241-248.

CPRA, 2012. Louisiana's Comprehensive Master Plan for a Sustainable Coast. Coastal Protection and Restoration Authority of Louisiana, Baton Rouge, LA.

Dalrymple, R.A., Kirby, J.T., Hwang, P.A., 1984. Wave refraction due to areas of energy dissipation. Journal of Waterway, Port, Coastal, and Ocean Engineering 110, 67-79.

Dixon, A.M., Leggett, D.J., Weight, R.C., 1998. Habitat creation opportunities for landward coastal re-alignment: Essex case study. Journal of Chartered Institution of Water and Environmental Management 12, 107-112.

Dubi, A., Tørum, A., 1996. Wave energy dissipation in kelp vegetation. Proceedings of the 25th International Conference on Coastal Engineering. ASCE, New York, NY, pp. 2626-2639.

Gedan, K.B., Kirwan, M.L., Wolanski, E., Barbier, E.B., Silliman, B.R., 2011. The present and future role of coastal wetland vegetation in protecting shorelines: an answering recent challenges to the paradigm. Climatic Change 106, 7-29.

Hasselmann, K., Collins, J., 1968. Spectral dissipation of finite-depth gravity waves due to turbulent bottom friction. Journal of Marine Research 26, 1-12.

Jadhav, R.S., Chen, Q., in review. Wave attenuation by salt marsh vegetation during tropical cyclone. Journal of Geophysical Research.

Kobayashi, N., Raichlen, A.W., Asano, T., 1993. Wave attenuation by vegetation. Journal of Waterway, Port, Coastal, and Ocean Engineering 119, 30-48.

Lopez, J.A., 2009. The multiple lines of defense strategy to sustain coastal Louisiana. Journal of Coastal Research 186-197 (Special Issue 54 - Geologic and Environmental Dynamics of the Pontchartrain Basin (FitzGerald \& Reed)).

Løvås, S.M., Tørum, A., 2001. Effect of kelp Laminaria hyperborea upon sand dune erosion and water particle velocities. Coastal Engineering 44, 37-63.

Lövstedt, C.B., Larson, M., 2010. Wave damping in reed: field measurements and mathematical modeling. Journal of Hydraulic Engineering 136 (4), 222-233.

Lowe, R., Koseff, J.R., Monismith, S.G., 2005. Oscillatory flow through submerged canopies: 1.Velocity structure. Journal of Geophysical Research 110, C10016. http:// dx.doi.org/10.1029/2004JC002788.

Lowe, R., Falter, J., Koseff, J.R., Monismith, S.G., Atkinson, M., 2007. Spectral wave flow attenuation within submerged canopies: implications for wave energy dissipation. Journal of Geophysical Research 112, C05018. http://dx.doi.org/10.1029/2006JC003605.

Mazda, Y., Magi, M., Ikeda, Y., Kurokawa, T., Asano, T., 2006. Wave reduction in a mangrove forest dominated by Sonneratia sp. Wetlands Ecology and Management 14, 365-378.

Mendez, F.J., Losada, I.J., 2004. An empirical model to estimate the propagation of random breaking and non-breaking waves over vegetation fields. Coastal Engineering 51, 103-118.

Mendez, F., Losada, I., Losada, M., 1999. Hydrodynamics induced by wind waves in a vegetation field. Journal of Geophysical Research 104 (C8), 18,383-18,396.

Möller, I., 2006. Quantifying salt marsh vegetation and its effect on wave height dissipation: results from a UK East coast saltmarsh. Estuarine, Coastal and Shelf Science 69, 337-351.

Möller, I., Spencer, T., 2002. Wave dissipation over macro-tidal salt marshes: effects of marsh edge typology and vegetation change. Journal of Coastal Research SI36, 506-521.

Möller, I., Spencer, T., French, J.R., Leggett, D., Dixon, M., 1999. Wave transformation over salt marshes: a field and numerical modelling study from North Norfolk, England. Estuarine, Coastal and Shelf Science 49, 411-426.

Morison, J.R.M., O'Brien, M.P., Johnson, J.W., Schaaf, S.A., 1950. The force exerted by surface waves on piles. Petroleum Transactions AWME 189, 149-154.

Mullarney, J., Henderson, S., 2010. Wave-forced motion of submerged single-stem vegetation. Journal of Geophysical Research 115, C12061. http://dx.doi.org/10.1029/ 2010JC006448.

Neumeier, U., Amos, C.L., 2006. The influence of vegetation on turbulence and flow velocities in European salt-marshes. Sedimentology 53 (2), 259-277.

Paul, M., Amos, C.L., 2011. Spatial and seasonal variation in wave attenuation over Zostera noltii. Journal of Geophysical Research 116, C08019. http://dx.doi.org/ 10.1029/2010JC006797.

Quartel, S., Kroon, A., Augustinus, P.G.E.F., Van Santen, P., Tri, N.H., 2007. Wave attenuation in coastal mangroves in the Red River Delta, Vietnam. Journal of Asian Earth Sciences 29 (4), 576-584.

Riffe, K.C., Henderson, S.M., Mullarney, J.C., 2011. Wave dissipation by flexible 821 vegetation. Geophysical Research Letters 38, L18607. http://dx.doi.org/10.1029/ 2011 GL048773 (5 pp.).

Sánchez-González, J., Sánchez-Rojas, V., Memos, C., 2011. Wave attenuation due to Posidonia oceanica meadows. Journal of Hydraulic Engineering 49 (4), 503-514.

Sarpkaya, T., 1976. Vortex shedding and resistance in harmonic flow about smooth and rough circular cylinders at high Reynolds numbers. Naval Post Graduate School Technical Report No: NPS-59L76021, Monterey CA, USA.

Stratigaki, V., Manca, E., Prinos, P., Losada, I.J., Lara, J.L., Sclavo, M., Amos, C.L., Cáceres, I., Sánchez-Arcilla, A., 2011. Large-scale experiments on wave propagation over Posidonia oceanica. Journal of Hydraulic Engineering 49 (Suppl. 1), 31-43.

Suzuki, T., Zijlema, M., Burger, B., Meijer, M.C., Narayan, S., 2011. Wave dissipation by vegetation with layer schematization in SWAN. Coastal Engineering 59, 64-71.

Tanino, Y., Nepf, H.M., 2008. Laboratory investigation of mean drag in a random array of rigid, emergent cylinders. Journal of Hydraulic Engineering 134 (1), 34-41. 